\title{
MiR-142-3p Overexpression Increases Chemo-Sensitivity of NSCLC by Inhibiting HMGB1-Mediated Autophagy
}

\author{
Yuqing Chen ${ }^{\mathrm{a}}$ Xin Zhou ${ }^{\mathrm{a}}$ Jianou Qiao ${ }^{\mathrm{b}}$ Aihua Bao ${ }^{\mathrm{a}}$ \\ aDepartment of Respiratory, Shanghai General Hospital, Shanghai Jiao Tong University School of

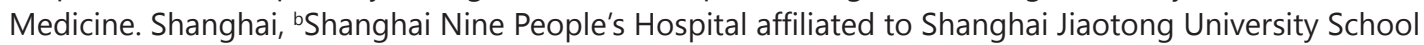 \\ of Medicine Shanghai, China
}

\section{Key Words}

miR-142-3p • HMGB1 • Autophagy • NSCLC • PI3K/Akt/mTOR pathway

\begin{abstract}
Background: Non-small-cell lung cancer (NSCLC) is a deadly cancer with high mortality rate. Drug resistance represents a main obstacle in NSCLC treatment. High mobility group box-1 (HMGB1) protein promotes drug resistance in NSCLC cells by activating protective autophagy. Methods: In the current study, we investigated the regulatory role of microRNA-142-3p (miR142-3p) in HMGB1-mediated autophagy of NSCLC cells and its impact on drug resistance of NSCLC in vitro and in vivo. HMGB1 was identified as a putative target gene of miR-142-3p by in silico analysis. Our luciferase reporter assay results confirmed that miR-142-3p directly targets the 3'-UTR of HMGB1 in NSCLC cells. Results: MiR-142-3p overexpression suppressed while miR-142-3p knockdown increased HMGB1 mRNA and protein expression. Starvation induced HMGB1 expression and activated autophagy in NSCLC cells. The starvation-induced autophagy was inhibited by miR-142-3p overexpression or HMGB1 knockdown. Moreover, miR-142$3 p$ overexpression or HMGB1 knockdown increased PI3K, Akt, and mTOR phosphorylation. Inhibition of PI3K or mTOR restored starvation-induced autophagy inhibited by miR-142-3p overexpression or HMGB1 knockdown. Conclusions: These results demonstrated that miR142-3p regulates starvation-induced autophagy of NSCLC cells by directly downregulating HMGB1 and subsequently activating the PI3K/Akt/mTOR pathway. Further, miR-142-3p overexpression inhibited anticancer drug-induced autophagy and increased chemo-sensitivity of NSCLC in vitro and in vivo. These findings shed light on the therapeutic potential of miR$142-3 p$ in combating acquired NSCLC chemo-resistance.

\section{Introduction}

Non-small-cell lung cancer (NSCLC) is one of the most deadly cancers worldwide [1]. Drug resistance, intrinsic or acquired, limits the efficacy of conventional chemotherapy and biological therapies, and thus represents a major obstacle in NSCLC treatment [2]. 
MicroRNAs (miRNAs) are small noncoding RNAs that downregulate target gene expression via base-pairing with complementary sequences within mRNA molecules, causing mRNA degradation or translational repression [3]. MiRNAs regulate a wide array of cell functions such as proliferation, differentiation, migration, and apoptosis, and are implicated in various pathological conditions including tumorigenesis [4-7]. Accumulating evidence also suggests that dysregulation of specific miRNAs may be involved in the acquisition of drug resistance of a number of cancers, particularly lung cancer $[8,9]$. Identifying miRNAs implicated in NSCLC and understanding the mechanisms by which they regulate NSCLC tumorigenesis and/or drug resistance is of critical importance in seeking to improve NSCLC treatment.

MiRNA-142-3p (miR-142-3p) is dyregulated in many malignancies and can function as a tumor suppressor or oncogene by regulating tumor cell proliferation, migration, and invasion [10-18]. With regard to NSCLC, current evidence remains somewhat controversial and inconclusive. Increased circulating levels of miR-142-3p were found to be associated with poor clinical outcome of NSCLC and a high risk of relapse in early-stage lung adenocarcinoma [19, $20]$. Additionally, miR-142-3p has been shown to repress TGF- $\beta$-mediated growth inhibition through downregulation of TGF $\beta R 1$ in NSCLC [21], These findings suggest an oncogenic role of miR-142-3p in this malignancy. However, another study has reported that miR-142$3 p$ was downregulated in NSCLC tissues and cell lines, and miR-142-3p overexpression inhibited NSCLC cell proliferation and induced cell apoptosis in vitro, supporting an antitumor function of miR-142-3p in NSCLC[18]. Taken together, the functions of miR-142-3p in NSCLC are likely context-dependent and require further investigation.

Autophagy is a highly regulated catabolic process that allows the orderly degradation of unnecessary or dysfunctional cellular components, for the maintenance of cellular homeostasis. Autophagy plays a context-dependent role in the development and progression of cancer [22, 23]. It can promote tumor cell death and thereby function as a tumor suppressor. Nevertheless, under stress conditions such as hypoxia and nutrient starvation, autophagy can also function as a cytoprotective mechanism that promotes cancer cell survival and resistance to chemotherapy, radiation therapy, and immunotherapy [22, 24]. Indeed, studies have shown that autophagy contributes to NSCLC chemo-resistance under hypoxic conditions [25]. High mobility group box-1 (HMGB1) protein, a DNA-binding protein that regulates cell death and survival, plays a central role in carcinogenesis [26]. Intriguingly, recent studies indicate that HMGB1 also promotes chemo-resistance of a number of cancer cells including NSCLC by activating protective autophagy [27-31]. In particular, suppression of HMGB1 sensitizes NSCLC cells to docetaxel and cisplatin by inhibiting autophagy and increasing apoptosis [30,31]. Thus, targeting HMGB1-mediated autophagy might have clinical significance in NSCLC treatment.

In this study, we investigated the regulatory role of miR-142-3p in HMGB1-mediated autophagy as well as chemo-resistance of NSCLC in vitro and in vivo. MiR-142-3p has recently been reported to directly target HMGB1 in NSCLC[18]. Our results demonstrated that miR142-3p directly regulates HMGB1 in NSCLC cells. MiR-142-3p overexpression inhibits starvation-induced autophagy of NSCLC cells by directly downregulating HMGB1 and subsequently activating the PI3K/Akt/mTOR pathway. Further, miR-142-3p overexpression inhibits anticancer drug-induced autophagy and increases chemo-sensitivity of NSCLC in vitro and in vivo.

\section{Materials and Methods}

Cell culture and reagents

Normal human bronchial epithelial (HBE) cells and human A549 NSCLC cells were purchased from Cell Bank of the Chinese Academy of Sciences (Shanghai, China). Cells were grown in RPMI 1640 medium (HyClone, USA) supplemented with 10\% FBS, 1\% L-glutamine, and 1\% Penicillin/Streptomycin (Invitrogen, USA) at $37^{\circ} \mathrm{C}, 5 \% \mathrm{CO}_{2}$ in a humidified incubator. MiR-142-3p mimic (miR-142-3p) and antagomiR (antimiR-142-3p), and two scramble miRNAs used as negative controls (miR-NC for miR-142-3p; anti-miR-

\section{KARGER}


Chen et al.: MiR-142-3p Increases Chemo-Sensitivity of NSCLC

NC for anti-miR-142-3p) were synthesized at GenePharma (Shanghai, China). SiRNA against HMGB1 was purchased from Genechem (Shanghai, China). The PI3K inhibitor LY294002 was purchased from Cell Signaling Technology Inc. (USA). Adriamycin (ADM), cisplatin (DDP), and the mTOR inhibitor rapamycin were all from Sigma-Aldrich (USA).

Real-time quantitative reverse transcription-polymerase chain reaction ( $q R T-P C R$ )

Total RNA was extracted from A549 cells using an RNeasy Mini Kit (Qiagen, USA) following manufacturer's instructions. The RT primers for miRNA cDNA synthesis are: MiR-142-3p, GTCGTATCCAGTGCAGGGTCCGAGGTATTCGCACTGGATACGACTCCATAAA; U6, CGAGCACAGAATCGCTTCACGAATTTGCGTGTCAT. The primers for mRNA detection are: miR-1423p, 5'-GTCGTATCCAGTGCAGGG-3'(forward) and 5'-CGACGTGTAGTGTTTCCTA-3' (reverse); HMGB1, 5'-GATGGGCAAAGGAGATCCTA-3' (forward) and 5'-CTTGGTCTCCCTTTGGGG-3' (reverse). Real-time quantitative PCR (qRT-PCR) was performed using a SYBR green qPCR SuperMix-UDG kit (Life Technologies, USA) on an ABI PRISM 7300 system (Applied Biosystems). Ct values of HMGB1 mRNA were normalized to $\beta$-actin. Relative expression was calculated using the ${ }^{\Delta \Lambda} C t$ method.

\section{Western blot analysis}

Cell lysates and tumor tissue homogenates (50 $\mathrm{\mu g}$ per sample) were subjected to $10 \%$ sodium dodecyl sulfate-polyacrylamide gel electrophoresis (SDS-PAGE) and transferred to polyvinylidene fluoride (PVDF) membranes (Millipore, USA). After blocking in 5\% nonfat milk for $1 \mathrm{~h}$, the membranes were incubated with anti-HMGB1, anti-p62, anti-LC3, anti-PI3K, anti-p-PI3K, anti-mTOR, anti-p-mTOR, anti-Akt, anti-p-Akt, or anti- $\beta$-actin at $4{ }^{\circ} \mathrm{C}$ overnight. After washing with PBS, the membranes were incubated with horseradish peroxidase-conjugated secondary antibody for $2 \mathrm{~h}$ at room temperature. Signals were detected using an ECL detection system (Bio-Rad, USA). All primary antibodies except anti- $\beta$-actin were from Cell Signaling Technology Inc. Anti- $\beta$-actin was purchased from Sigma-Aldrich.

\section{Luciferase reporter assay}

The putative miR-142-3p binding site in the 3'-UTR of HMGB1 was predicted using miRanda-mirsVR (http://www.microrna.org/microrna/home.do). A fragment containing the putative miR-142-3p binding site was amplified and subcloned into the pGL3 plasmid to generate the pGL3-HMGB1 3'-UTR-wild type. A fragment containing the mutant binding site with six-base changes was created using the Quick-Change Site Directed Mutagenesis Kit (Invitrogen) and subcloned into the pGL3 plasmid to generate the pGL3-HMGB1 3'-UTR-mutant. A549 cells were seeded in 96-well plates (1,000 cells/well) and cultured overnight. On the next day, the cells were co-transfected with $1.5 \mathrm{ng}$ of pGL3-HMGB1 3'-UTR-wild type/pGL3-HMGB1 3'-UTRmutant and with $100 \mathrm{nM}$ of either miR-142-3p or miR-NC using Lipofectamine 2000 (Invitrogen). Luciferase activity was detected $72 \mathrm{~h}$ after transfection using the Dual-Luciferase Reporter Assay Kit (Promega, USA) on a TD20/20 Luminometer (Turner Designs).

\section{Detection of autophagosomes with GFP-LC3 labeling}

A549 cells were transfected with GFP-LC3 cDNA using Lipofectamine 2000 reagent (Invitrogen) according to the supplier's protocol. The transfected cells were seeded in six-well plates $\left(5 \times 10^{5}\right.$ cells/well $)$ and cultured overnight. On the next day, cells were transfected with $100 \mathrm{nM}$ miR-142-3p or miR-NC for $72 \mathrm{~h}$ using Lipofectamine 2000 (Invitrogen). Cells were subsequently subjected to $20 \mathrm{~min}$ starvation in serum-free Hank's balanced salt solution (HBSS). GFP-LC3-labeled vacuoles (autophagosomes) were counted under a fluorescence microscope (Olympus IX70). To assess drug-induced autophagy, A549 cells labeled with GFP-LC3 weretransfected with100nMmiR-142-3p ormiR-NCfor $24 \mathrm{~h}$. ThecellsweresubsequentlyincubatedwithADM(5 M), DDP $(20 \mu \mathrm{M})$ or vehicle alone (control) for $48 \mathrm{~h}$ and subjected to autophagosome detection by fluorescence imaging.

\section{Cell proliferation assay}

A549 cells transfected with $100 \mathrm{nM}$ miR-142-3p or miR-NC for $24 \mathrm{~h}$ were seeded in 96-well plates $\left(5 \times 10^{3}\right.$ cells/well) and treated with ADM $(5 \mu \mathrm{M})$, DDP $(20 \mu \mathrm{M})$ or vehicle alone (control) for $48 \mathrm{~h}$. Cell viability was examined by the MTT-Cell Titer 96 AQueous One Solution Cell Proliferation Assay (Invitrogen) following manufacturer's instructions.

\section{KARGER}


Apoptosis assays

Cell apoptosis was assessed by measuring the activation of caspase- 3 and TUNEL staining. The caspase- 3 activity was determined using a caspase-3 assay kit from BioVision Inc. (Mountain View, CA, USA) following manufacturer's instructions. TUNEL assays were performed using the in situ Cell Death Detection Kit (Roche Diagnostics GmbH, Mannheim, Germany) following manufacturer's instructions. The total number of cells in a given area was determined by DAPI staining. The apoptotic index is presented as the percentage of TUNEL-positive cells.

\section{Preparation of stable cell lines}

A549 cells stably expressing miR-142-3p or miR-NC were used to generate NSCLC xenograft model. In brief, A549 cells were infected with lentiviruses carrying miR-142-3p or miR-NC (Genomeditech Inc., Shanghai, China) for $48 \mathrm{~h}$. The cells were subsequently selected by puromycin for 1 weeks for stable expression of miR-142-3p or miR-NC.

\section{Animal experiments}

Male nude mice ( 5 weeks old) were purchased from the Shanghai Laboratory Animal Company (SLAC, Shanghai, China). A549 cells stably expressing miR-142-3p or miR-NC $\left(5 \times 10^{6}\right.$ cells in $100 \mu$ l phosphatebuffered saline (PBS)) were subcutaneously injected into the right flank of mice ( $n=8$ per group). After one week, DDP (2.0 mg/kg) or ADM $(2.0 \mathrm{mg} / \mathrm{kg})$ was administered via intraperitoneal injection three times a week for three weeks. The tumor volume was measured once every four days using the modified ellipse volume formula: volume $=($ height*width*depth $) / 2$. At the end of treatment, the mice were sacrificed and the tumors were collected. The tumor tissues were subjected to immunohistological analysis by hematoxylin and eosin (H\&E), proliferating cell nuclear antigen (PCNA), and TUNEL staining. Tumoral HMGB1, LC3 and p62 levels were determined by western blot analysis.

\section{Statistical analysis}

All data are presented as means \pm standard deviation (SD). Two-way ANOVA was applied to interpret the differences between treatment groups. Differences with a $P$ value $<0.05$ were considered statistically significant.

\section{Results}

HMGB1 is a direct target of miR-142-3p in human NSCLC cells

We first examined the expression of miR-142-3p in normal human bronchial epithelium (HBE) cells and A549 human NSCLC cells by qRT-PCR. As shown in Figure 1A, the expression of miR-142-3p in A549 cells was significantly lower than that in HBE cells. This was consistent with previous findings of miR-142-3p downregulation in NSCLC[18]. MiR-142-3p has been reported to directly regulate HMGB1 in human lung cancer cells[18]. In this study, we independently identified a putative miR-142-3p binding site in the 3'-UTR of HMGB1 (Fig. 1B) using miRanda-mirsVR (http://www.microrna.org/microrna/home.do). To find out whether HMGB1 is a bona fide target of miR-142-3p in NSCLC cells, we prepared luciferase reporter expression systems comprising the 3'-UTR of human HMGB1 containing the putative miR-142-3p binding site (wild type or mutant) (Fig. 1B). Transient co-transfection of the reporter construct containing the wild type 3'-UTR with miR-142-3p into A549 cells resulted in approximately $60 \%$ loss of luciferase reporter activity $(P<0.01$, Fig. $1 C)$. However, miR-142-3p co-transfection did not repress the luciferase activity when the wild type 3'-UTR was replaced with the mutant 3'-UTR in the luciferase reporter system (Fig. 1C). We next examined whether miR-142-3p could regulate endogenous HMGB1 expression in NSCLC cells. Our qRT-PCT results showed that miR-142-3p expression was significant elevated by miR-142-3p transfection while reduced by anti-miR-142-3p transfection (Fig. 1D). We detected decreased HMGB1 mRNA and protein levels in A549 cells transfected with miR142-3p, meanwhile, increased HMGB1 levels were found in A549 cells transfected with antimiR-142-3p $(P<0.01$, Fig. $1 E, 1 F)$. Taken together, these results confirmed that miR-142-3p directly downregulates HMGB1 in NSCLC cells by targeting the 3'-UTR of HMGB1. 
Fig. 1. MiR-142-3p directly regulates HMGB1 expression in NSCLC cells by targeting the 3 '-UTR of HMGB1. A) The miR-142-3p levels in A549 and HBE cells by TaqMan qRT-PCR. $\mathrm{n}=$ $3,{ }^{* *} P<0.01$ vs. HBE. $B$ ) Human miR$142-3 p$ sequence and the seed sequences of the wild type (HMGB1 3'UTR WT) or mutant 3'-UTR (HMGB1 3'-UTR MUT) of human HMGB1. C) A549 cells were co-transfected with pGL3-HMGB1 3'-UTR-WT/pGL3-HMGB1 3'-UTR-MUT and miR-142-3p or miR-NC for 72 h. Luciferase activity was detected using the Dual-Luciferase Reporter Assay Kit from Promega. $\mathrm{n}=3,{ }^{* *} P<0.01$ vs. pGL3-HMGB1 3'-UTR-WT + miR-NC. $D-F$ ) A549 cells were transfected with miR-142-3p or anti-miR-142-3p for $72 \mathrm{~h}$. MiR-NC and anti-miR-NC were used as negative controls. The miR-142-3p levels were determined by TaqMan qRT-PCR $(D)$; and the HMGB1 protein and mRNA levels were determined by western blot analysis $(E)$ and qRT-PCR $(F)$, respectively. $\mathrm{n}=3{ }^{* *} P<0.01$.

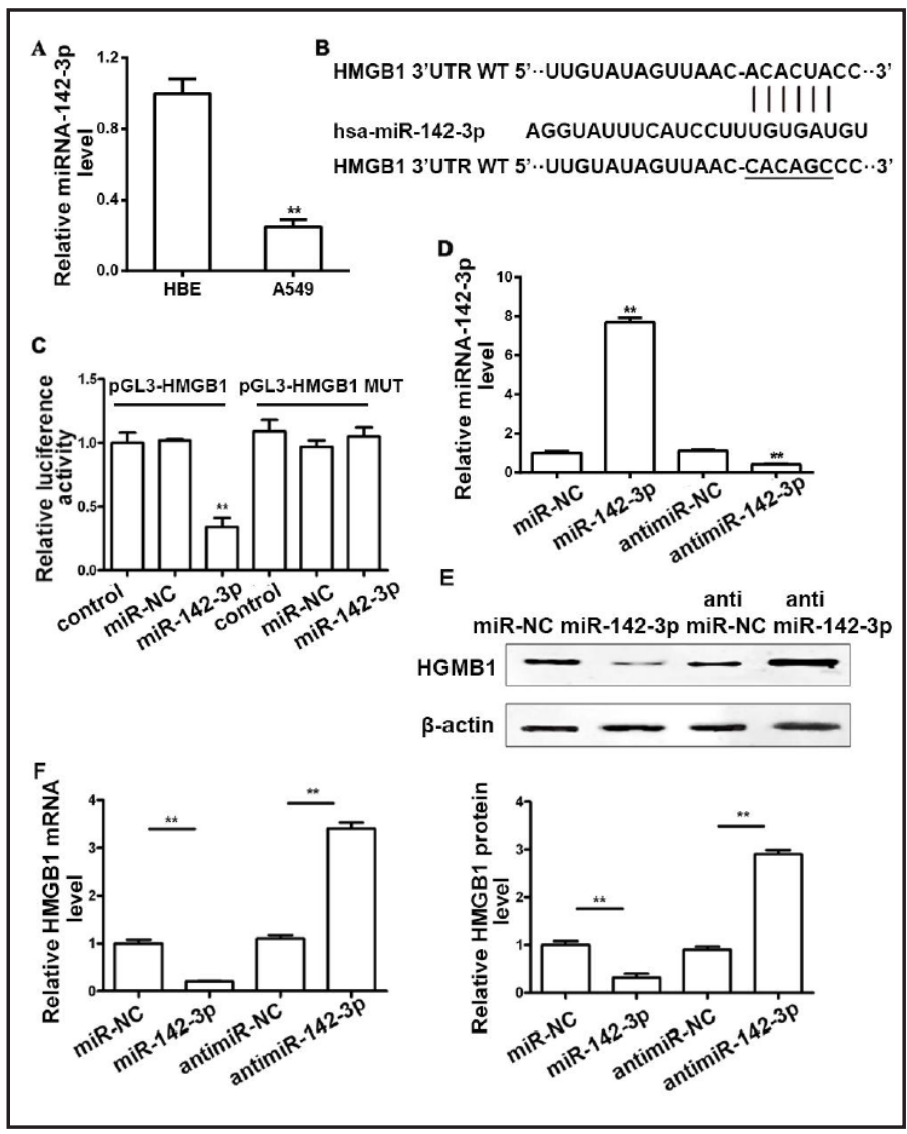

MiR-142-3p overexpression inhibits starvation-induced autophagy of human NSCLC cells

It has been reported that HMGB1 knockdown by RNA interference inhibits autophagy and promotes chemo-resistance in NSCLC cells [30,31]. Based on these previous findings, we speculated that miR-142-3p could regulate NSCLC cell autophagy by downregulating HMGB1. To test this, we investigated the effects of miR-142-3p overexpression on starvationinduced autophagy in human NSCLC cells. Autophagy is characterized by the formation of autophagosomes and autolysosomes. Microtubule-associated protein-1 light chain 3 (LC3), a major constituent of the autophagosome, is a reliable marker of autophagosomes. In this study, we used NSCLC cells transfected with green fluorescent protein (GFP)-tagged LC3 (GFP-LC3) to detect autophagosome formation. Nutrient starvation for $20 \mathrm{~min}$ in serumfree HBSS medium significantly induced the formation of GFP-LC3-labeled vacuoles in miR-NC-transfected but not miR-142-3p-transfected A549 cells (Fig. 2A), indicating that miR-142-3p overexpression suppresses starvation-induced autophagosome formation. To further characterize autophagy, we determined the conversion of LC3-I to LC3-II and the protein level of p62 using western blot analysis. The conversion of soluble LC3-I to lipid bound LC3-II is associated with the formation of autophagosomes during autophagy. P62 is an LC3-binding protein that is degraded through the autophagy-lysosomal pathway [32]. Our results showed that nutrient deprivation for $4 \mathrm{~h}$ resulted in increased LC3-II to LC3-I ratio as well as decreased p62 level. These autophagy-associated changes were attenuated by miR-142-3p overexpression $(P<0.01$, Fig. $2 B, 2 C)$, further supporting that miR-142-3p overexpression inhibits starvation-induced NSCLC cell autophagy. Interestingly, miR-142$3 p$ overexpression also led to decreased LC3-II/LC3-I ratio and increased p62 level in nonstarved cells $(P<0.05$, Fig. $2 B, 2 C)$, suggesting that miR-142-3p overexpression protects NSCLC cells from autophagy even under normal, nonstressed conditions. 


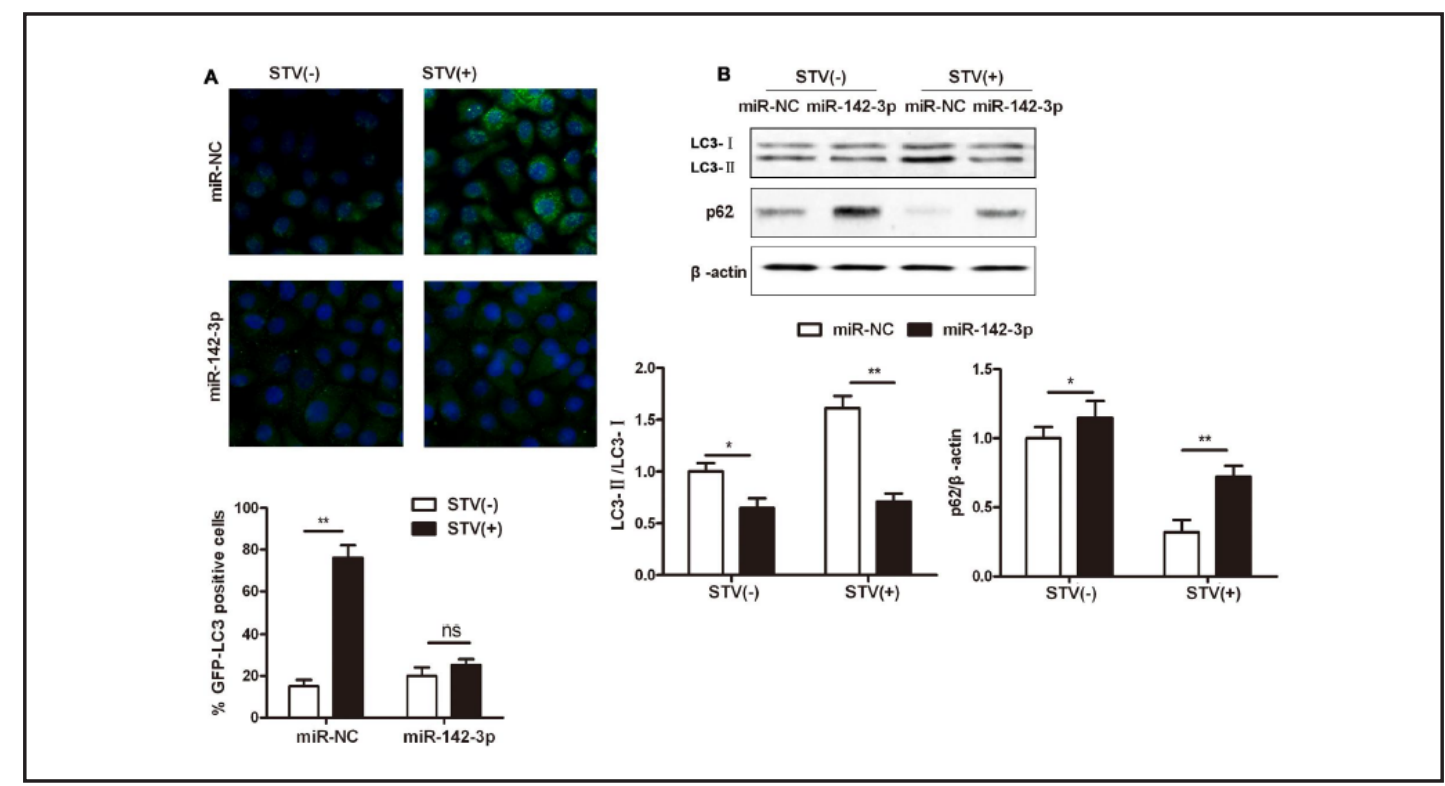

Fig. 2. MiR-142-3p overexpression inhibits starvation-induced autophagy of NSCLC cells. A) A549 cells labeled with GFP-LC3 were transfected with $100 \mathrm{nM}$ miR-142-3p or miR-NC for $72 \mathrm{~h}$ and subjected to 20 min nutrient starvation. Representative fluorescent microscopic images showing GFP-LC3-labeled vacuoles (dots) and the percentage of cells with detectable GFP-LC3 dots are shown. STV(-), without starvation; $\operatorname{STV}(+)$, with starvation. $\mathrm{n}=3,{ }^{* *} P<0.01$, ns $=$ nonspecific. Magnification, $\left.\times 400 . B\right)$ A549 cells were transfected with $100 \mathrm{nM}$ miR-142-3p or miR-NC for $72 \mathrm{~h}$ and subsequently starved for $4 \mathrm{~h}$. The LC3-I, LC3-II and p62 protein levels were determined by western blot analysis. $\beta$-actin was used as loading control. $n=3,{ }^{*} P<0.05$, $* * P<0.01$.

MiR-142-3p regulates starvation-induced autophagy of human NSCLC cells through HMGB1

To reveal the role of endogenous miR-142-3p in NSCLC cell autophagy, we examined the effects of miR-142-3p knockdown. In contrast to miR-142-3p overexpression, knockdown of endogenous miR-142-3p by anti-miR-142-3p transfection led to significantly increased LC3-I to LC3-II conversion and decreased p62 level in nutrient-deprived A549 cells (Fig. $3 A$ ). These results provided evidence that endogenous miR-142-3p in A549 cells functions to suppress starvation-induced autophagy. Interestingly, miR-142-3p knockdown also increased autophagic activity in nonstarved A549 cells (Fig. $3 A$ ), showing that endogenous miR-142-3p protects A549 cells from autophagy even under normal, nonstressed conditions. Importantly, these anti- and pro-autophagic effects of miR-142-3p overexpression and knockdown were accompanied by down- and up-regulation of HMGB1, respectively (Fig. $3 A$ ), and HMGB1 knockdown by si-HMGB1 transfection downregulated starvation-induced autophagy (Fig. 3B). These data supported that miR-142-3p inhibits starvation-induced autophagy of NSCLC cells by downregulating HMGB1.

MiR-142-3p overexpression inhibits starvation-induced autophagy of human NSCLC cells through the PI3K/Akt/mTOR pathway

The PI3K/Akt/mTOR pathway has been implicated in drug-induced autophagy in cancer cells [33-35]. In NSCLC, the anti-tumor drug platycodin-D (PD) has been shown to induce autophagy in A549 cells by inhibiting the PI3K/Akt/mTOR and activating the JNK and p38 MAPK signaling pathways [35]. In this study, we found that inhibition of starvationinduced autophagy by miR-142-3p overexpression or HMGB1 knockdown in A549 cells was accompanied by increased PI3K, Akt, and mTOR phosphorylation $(P<0.01$, Fig. $4 A)$. To confirm the involvement of the PI3K/Akt/mTOR pathway in the effects of miR-142-3p, 
Fig. 3. MiR-142-3p regulates starvation-induced autophagy of NSCLC cells through HMGB1. A) A546 cells were transfected with 100 nM miR-142-3p, miR-NC, anti-miR-142-3p, or anti-miR-NC for $72 \mathrm{~h}$ and subsequently subjected to $4 \mathrm{~h}$ starvation. The HMGB1, LC3-I, LC3-II, and p62 protein levels were determined by western blot analysis. $\beta$-Actin was used as loading control. control, untransfected cells; STV(-), without starvation; $\operatorname{STV}(+)$, with starvation. $\mathrm{n}=3$; ${ }^{*} P<0.05$, ${ }^{* *} P<0.01$ vs. control/STV(-); ${ }^{\#} P<0.05$, ${ }^{\# \#} P<0.01$ vs. control/ STV(+). B) A549 cells were transfected with $100 \mathrm{nM}$ miR142-3p, miR-NC, si-HMGB1, or siRNA-NC for $24 \mathrm{~h}$ in the starvation medium. The HMGB1, LC3-I, LC3-II, and p62 protein levels were determined by western blot analysis. control, untransfected cells incubated for $24 \mathrm{~h}$ in the starvation medium. $\mathrm{n}=3,{ }^{* *} P<0.01$ vs. control.

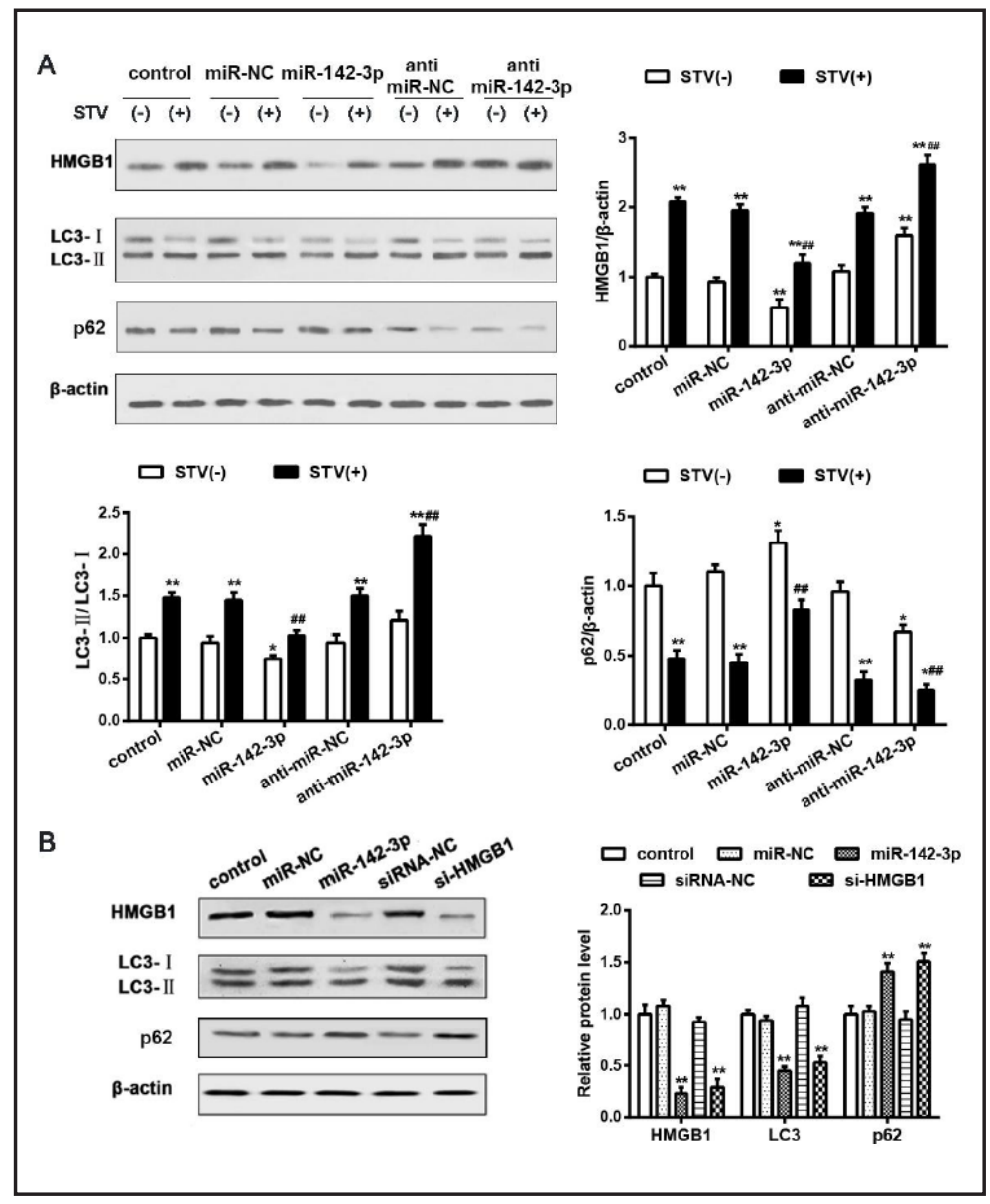

we tested the PI3K inhibitor LY294002 and the mTOR inhibitor rapamycin in A549 cells transfected with miR-142-3p or si-HMGB1. The results showed that blocking the PI3K/Akt/ mTOR signaling pathway with either LY294002 or rapamycin restored starvation-induced autophagy inhibited by miR-142-3p or si-HMGB1 transfection (Fig. 4B, 4C). LY294002 or rapamycin treatment, however, did not affect HMGB1 expression (Fig. 4B, 4C). Taken together, these results confirmed that miR-142-3p inhibits starvation-induced NSCLC cell autophagy by modulating the activity of the PI3K/Akt/mTOR pathway through its target HMGB1.

MiR-142-3p overexpression inhibits anticancer drug-induced autophagy of human NSCLC cells through HMGB1

Chemotherapeutic drugs such as cisplatin (DDP) can induce chemo-resistance of NSCLC cells by activating HMGB1-mediated protective autophagy [31]. HMGB1 silencing prevents drug-induced autophagy, and thereby restores chemo-sensitivity of NSCLC [31]. Having shown that miR-142-3p inhibits starvation-induced autophagy by downregulating HMGB1, we speculated that miR-142-3p might also inhibit drug-induced autophagy by the same mechanism. To investigate this hypothesis, we tested the effects of the anticancer drugs ADM and DDP. In alignment with previous findings [31], ADM and DDP induced HMGB1-mediated autophagy in A549 cells as indicated by increased HMGB1 expression along with increased autophagosome formation, LC3-I to LC3-II conversion, and p62 degradation $(P<0.01$, Fig $5 A, 5 B$ ). In alignment with our speculation, the HMGB1-mediated autophagy of A549 cells induced by ADM and DDP was inhibited by miR-142-3p overexpression $(P<0.01$, Fig 5A, 5B). 


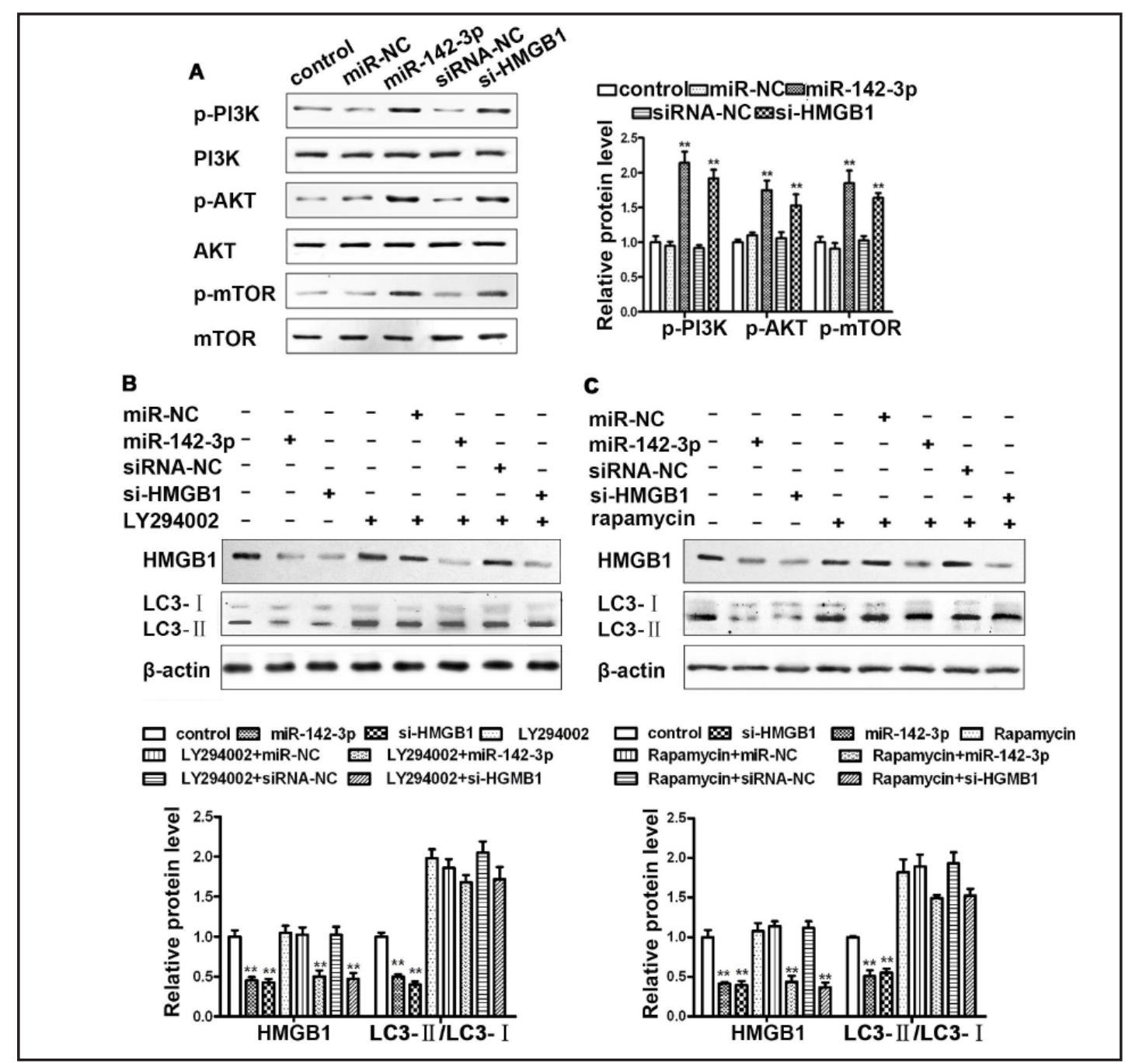

Fig. 4. MiR-142-3p overexpression and HMGB1 knockdown inhibit starvation-induced autophagy of NSCLC cells through the PI3K/Akt/mTOR pathway. A) A549 cells were transfected with $100 \mathrm{nM}$ miR-142-3p, miRNC, si-HMGB1, or siRNA-NC for $24 \mathrm{~h}$ in the starvation medium. The protein levels of PI3K, p-PI3K, Akt, p-Akt, mTOR, p-mTOR were determined by western blot analysis. control, untransfected cells incubated for $24 \mathrm{~h}$ in the starvation medium. $B, C$ ) A549 cells were transfected with $100 \mathrm{nM}$ miR-142-3p, miR-NC, si-HMGB1, or siRNA-NC for $24 \mathrm{~h}$ in the starvation medium containing the PI3K inhibitor LY294002 $(10 \mu \mathrm{M}) B$ ) or the mTOR inhibitor rapamycin $(10 \mu \mathrm{M}) C$ ). The protein levels of HMGB1, LC3-I, and LC3-II were determined by western blot analysis. $\beta$-Actin was used as loading control. control, untransfected cells incubated for $24 \mathrm{~h}$ in the starvation medium; $\mathrm{n}=3,{ }^{* *} P<0.01$ vs. control.

MiR-142-3p overexpression increases chemo-sensitivity of human NSCLC in vitro and vivo

Having shown that miR-142-3p overexpression inhibits anticancer drug-induced autophagy of NSCLC cells, we subsequently tested the effects of miR-142-3p overexpression on chemo-sensitivity of NSCLC in vitro and in vivo. ADM and DDP induced apoptotic cell death of A549 cells as indicated in decreased cell viability along with increased caspase-3 activity and TUNEL straining $(P<0.01$, Fig $6 A-C)$. We found that these cytotoxic effects of $\mathrm{ADM}$ and DDP were enhanced by miR-142-3p overexpression (Fig 6A-C). In an in vivo NSCLC xenograft model, intraperitoneal injection of ADM and DDP inhibited tumor growth of A549 cells implanted in male nude mice (Fig. 7A). PCNA and TUNEL staining of tumor tissues revealed decreased proliferation and increased apoptosis of tumor cells after ADM and DDP treatment (Fig. 7C). Importantly, ADM and DDP produced more potent cytotoxic effects in 

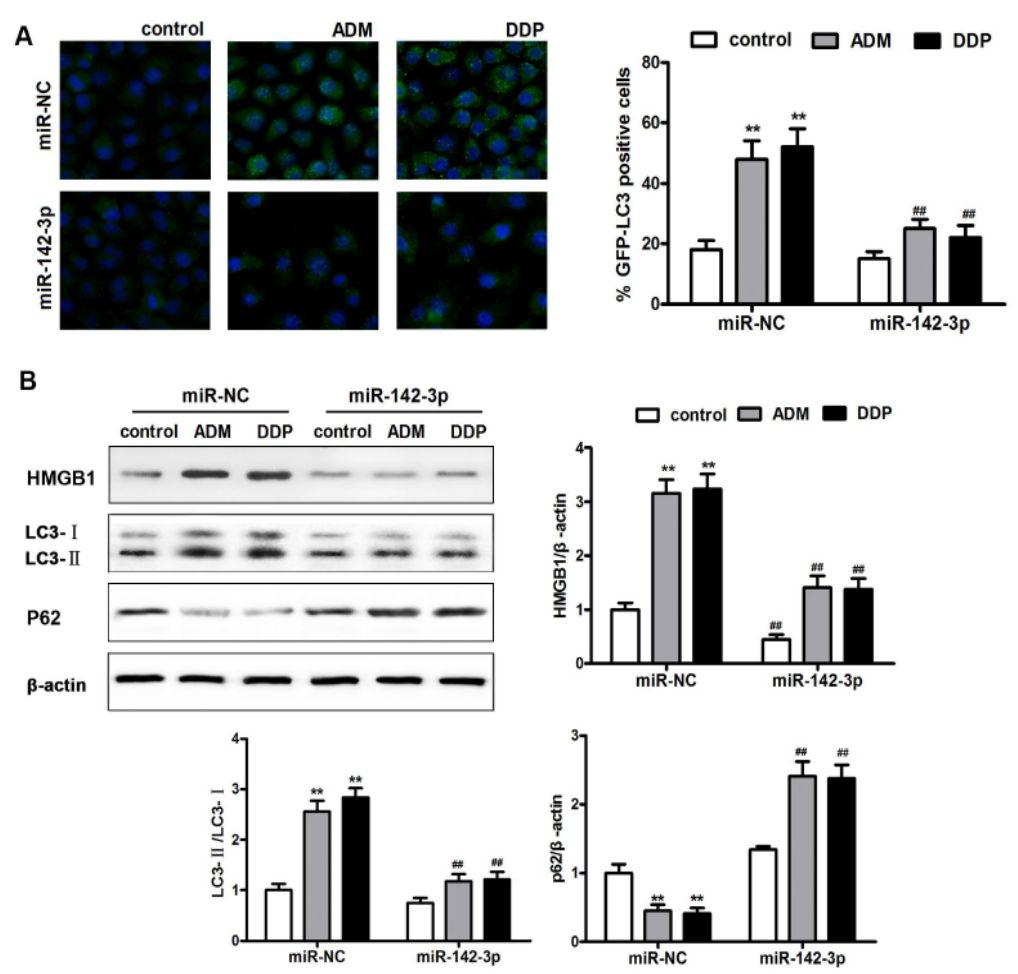

Fig. 5. MiR-142-3p overexpression inhibits drug-induced autophagy of NSCLC cells. A) A549 cells labeled with GFP-LC3 were transfected with $100 \mathrm{nM}$ miR-142-3p or miR-NC for $24 \mathrm{~h}$. The cells were subsequently incubated with ADM ( $5 \mu \mathrm{M})$, DDP $(20 \mathrm{M})$ or vehicle alone (control) for $48 \mathrm{~h}$. Representative fluorescent microscopic images showing GFP-LC3-labeled vacuoles (dots) and the percentage of cells with detectable GFP-LC3 dots are shown. B) A549 cells were incubated with ADM (5 $\mu$ M), DDP (20 M) or vehicle alone (control) for $24 \mathrm{~h}$. The cells were subsequently transfected with $100 \mathrm{nM}$ miR-142-3p or miR-NC for $48 \mathrm{~h}$. The protein levels of HMGB1, LC3-II, LC3-I, and p62 were determined by western blotting analysis. $\beta$-Actin was used as loading control. $\mathrm{n}=3,{ }^{* *} P<0.01$ vs. control/miR-NC, ${ }^{\# \#} P<0.01$ vs. miR-NC.

tumors overexpressing miR-142-3p in comparison with those overexpressing miR-NC (Fig. $7 A, 7 C$ ). Our western blot analysis data revealed increased tumoral HMGB1 expression and autophagic activity after ADM and DDP treatment (Fig. 7B). In comparison with tumors overexpression miR-NC, tumors overexpressing miR-142-3p exhibited lower HMGB1 expression and autophagic activity (Fig. $7 B$ ), suggesting that the chemo-sensitization effects of miR-142-3p overexpression are attributed to inhibition of drug-induced autophagy.

\section{Discussion}

Autophagy serves dual function in tumorigenesis, acting both as a tumor suppressor and a protector of cancer cell survival [36]. Excessive and sustained autophagy may lead to cell death and tumor shrinkage. However, in most contexts autophagy facilitates tumorigenesis by helping cancer cells to adapt and survive microenvironmental stresses such as hypoxia, nutrient deprivation, and cancer treatment (i.g. chemotherapy and radiation therapy) [37]. Extensive in vitro and in vivo studies have demonstrated that modulation of autophagy can be used as a therapeutic modality to enhance the efficacy of various anticancer regimens [38]. Currently, more than 30 clinical trials are investigating the therapeutic effects of autophagy inhibitors in combination with anticancer agents in treatment of various cancers including 
Fig. 6. $M i R-142-3 p$ overexpression increases the sensitivity of NSCLC to chemotherapy in vitro. A549 cells were transfected with $100 \mathrm{nM}$ miR142-3p or miR-NC for $24 \mathrm{~h}$. The cells were subsequently incubated with ADM $(5 \mu \mathrm{M})$, DDP (20 M) or vehicle alone (control) for 48 h. A) Cell viability determined by the MTT assay. B) Caspase-3 activity. C) Cell apoptosis assessed by TUNEL staining. $\mathrm{n}=$ 3; ${ }^{*} P<0.05,{ }^{*} P<0.01$ vs. control/miR-NC; ${ }^{\# \#} P<0.01$ vs. miR-NC.

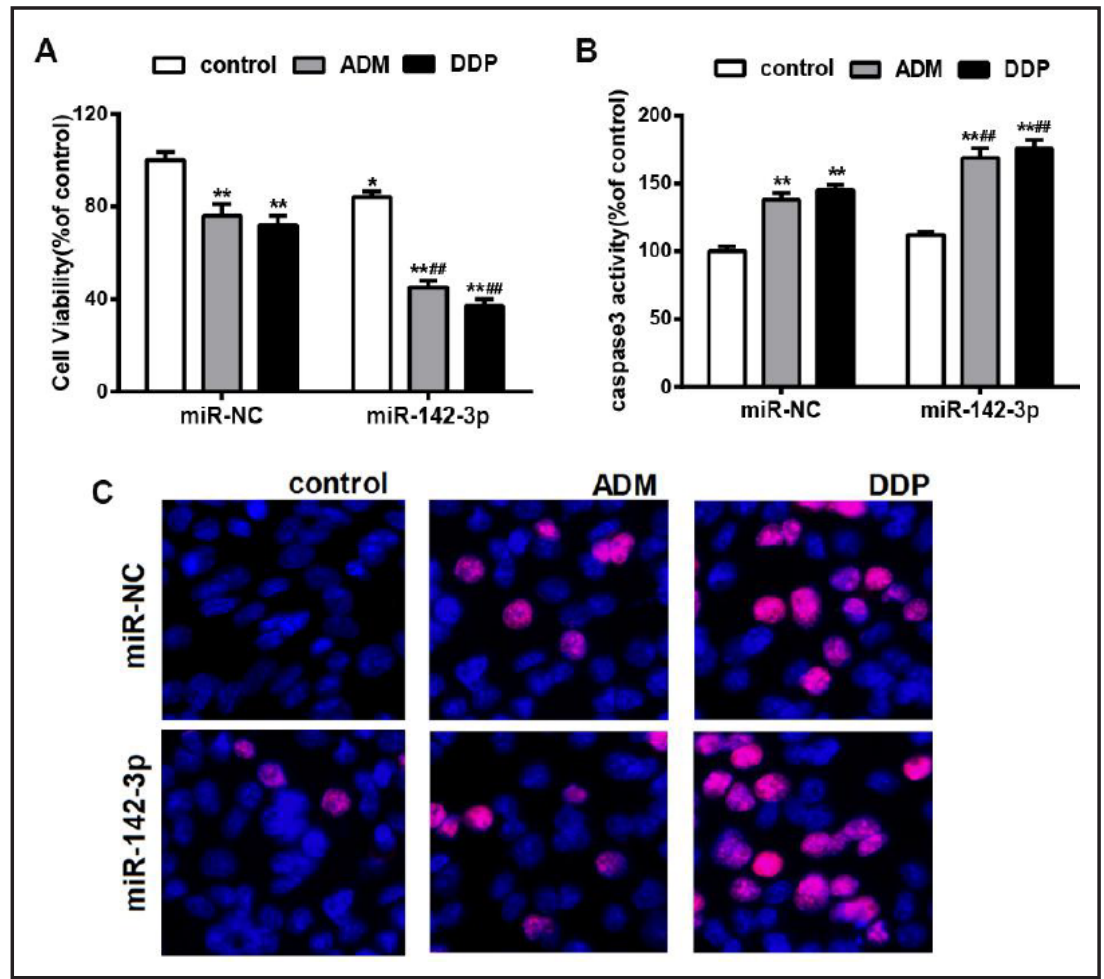

Fig. 7. MiR-142-3p overexpression increases the sensitivity of NSCLC to chemotherapy in vivo. A549 cells $\left(5.0 \times 10^{6}\right)$ stably expressing miR-142-3p or miR-NC were subcutaneously injected into the right flank of male nude mice. After one week, DDP (2.0 mg/kg) or ADM $(2.0 \mathrm{mg} / \mathrm{kg})$ was administered via intraperitoneal injection three times a week for three weeks. $A$ ) Tumor volume measured once every 4 days $(\mathrm{n}=6)$. $B)$ Tumoral HMGB1, LC3-II, LC3-I, and p62 levels determined by western blot analysis $(\mathrm{n}=3) . C) \mathrm{H} \& \mathrm{E}$ (original magnification, $\times 400$ ), PCNA(original magnification, $\times 200$ ), and TUNEL staining of tumor tissue sections (original magnification, $\times 200$ ). $* P<0.05, * * P<0.01$ vs. cont$\mathrm{rol} / \mathrm{miR}-\mathrm{NC}$; ${ }^{\#} P<0.05,{ }^{\# \#} P<0.01$ vs. miR-NC.

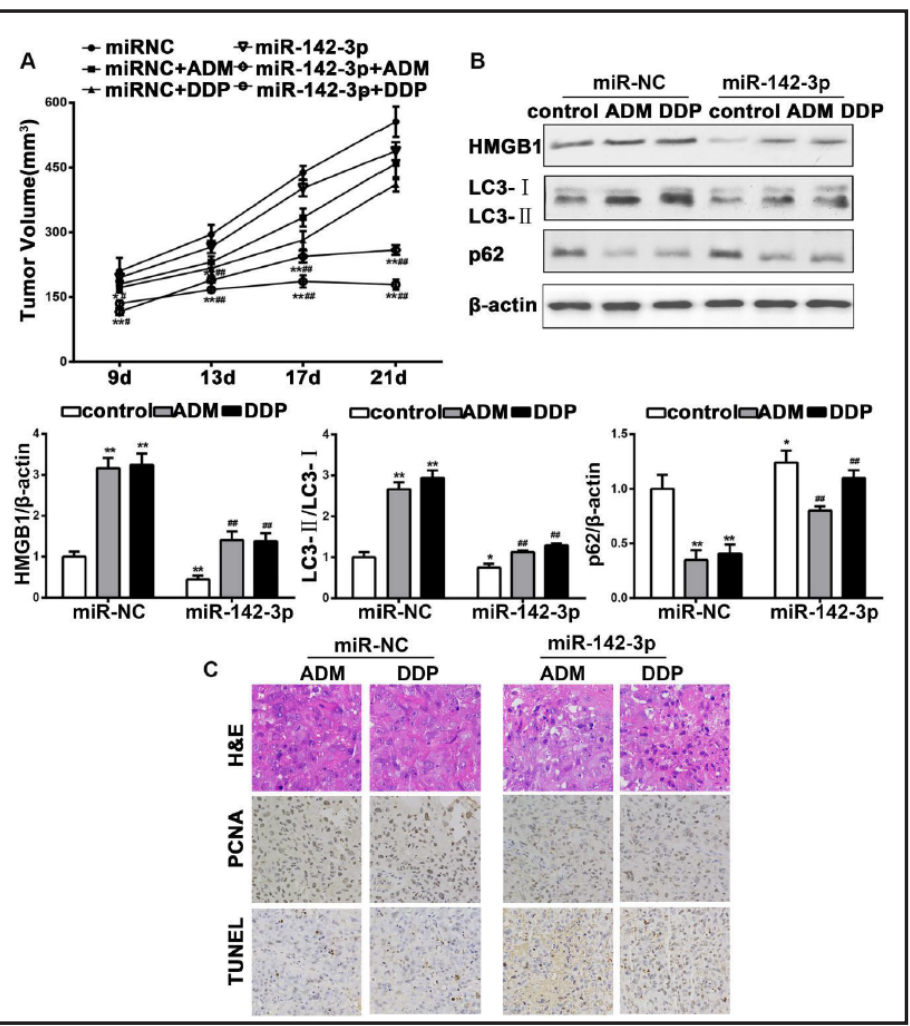

lung cancer [39]. Better understanding of autophagy regulation and its impact on treatment outcome will potentially allow us to identify novel therapeutic strategies to improve cancer treatment.

Many studies have shown that antitumor agents activate protective autophagy in NSCLC cells, causing acquired chemo-resistance [40-42]. Autophagy inhibition can overcome both 
the innate and acquired drug resistance of NSCLC, and thus represents a novel strategy to broaden the spectrum of NSCLC treatment [42-44]. In particular, autophagy inhibition has been exploited to improve the efficacy of small-molecule EGFR-tyrosine kinase inhibitors in the treatment of NSCLC patients $[39,44]$. Autophagy inhibitors in current NSCLC clinical trials include hydroxychloroquine (HCQ) and chloroquine (CQ) [39]. HCD and CD are US FDA-approved malaria drugs that inhibit autophagy at a late stage by blocking lysosomal acidification. In addition to HCD and CD, identification of novel and effective strategies to inhibit autophagy in NSCLC cells is needed.

A recent study has revealed that miR-143 inhibits NSCLC cell proliferation by targeting the autophagy gene, autophagy-related 2B [45]. HMGB1 is present at increased levels in NSCLC patients [46]. HMGB1-mediated autophagy has been reported to contribute to acquired NSCLC chemo-resistance [30,31]. In this study, we found that nutrient deprivation as well as the anticancer drug ADM or DDP induced HMGB1-mediated autophagy of NSCLC cells. This HMGB1-mediated autophagy was prevented by miR-142-3p overexpression. Our luciferase reporter assay results showed that miR-142-3p can directly downregulate HMGB1 in NSCLC cells by targeting its 3'-UTR. The PI3K/Akt/mTOR pathway, which is a key regulator of autophagy, has been implicated in HMGB1-mediated autophagy in leukemia cells [28]. In the current study, we found that the PI3K/Akt/mTOR pathway is involved in HMGB1-mediated autophagy induced by starvation, and miR-142-3p overexpression inhibits NSCLC cell autophagy by activating the PI3K/Akt/mTOR pathway through HMGB1. Finally, our results showed that miR-142-3p overexpression also inhibits anticancer drug-induced, HMGB1-mediated autophagy of NSCLC cells to increase the chemo-sensitivity of NSCLC in vitro and in vivo.

HMGB1 is involved in major DNA repair pathways and regulates cell death and survival [27]. Xiao and coworkers have reported that miR-142-3p directly targets HMGB1 to inhibit NSCLC cell proliferation and induce cell apoptosis [18], but the underlying mechanisms have not been elaborated. Our findings suggest that the in vitro anti-NSCLC properties of miR-142$3 p$ reported by Xiao et al. could be at least partially mediated by downregulation of HMGB1mediated autophagy. Findings from Xiao's and our work suggest that the downregulated miR-142-3p expression detected in NSCLC tissues and cells could be responsible for the upregulated HMGB1 expression in this malignancy, and the miR-142-3p/HMGB1 signaling likely contributes to both NSCLC tumorigenesis and chemo-resistance. Targeting the miR142-3p/HMGB1 signaling might be an effective strategy to find novel therapeutic agents for NSCLC.

\section{Funding}

This work was financially supported by National Nature Science Foundation of China (no. 81300005).

\section{Disclosure Statement}

The authors confirm that there are no conflicts of interest.

\section{References}

1 Vijayvergia N, Shah PC, Denlinger CS: Survivorship in non-small cell lung cancer: Challenges faced and steps forward. J Natl Compr Canc Netw 2015;13:1151-1161.

2 Spaans JN, Goss GD: Drug resistance to molecular targeted therapy and its consequences for treatment decisions in non-small-cell lung cancer. Front Oncol 2014;4:190.

-3 Bartel DP: Micrornas: Target recognition and regulatory functions. Cell 2009;136:215-233. 


\section{Cellular Physiology Cell Physiol Biochem 2017;41:1370-1382

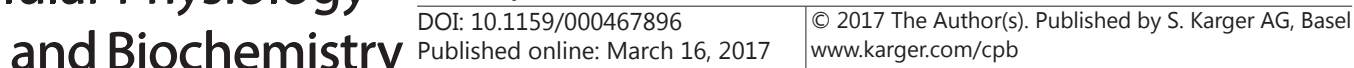

-4 Ivey KN, Srivastava D: Micrornas as developmental regulators. Cold Spring Harb Perspect Biol 2015; 7:a008144.

5 Chen Y, Gao Y, Zhang K, Li C, Pan Y, Chen J, Wang R, Chen L: MicroRNAs as Regulators of Cisplatin Resistance in Lung Cancer. Cell Physiol Biochem 2015;35:1381-1393.

6 He Z, Xia Y, Pan C, Ma T, Liu B, Wang J, Chen L, Chen Y: Up-Regulation of MiR-452 Inhibits Metastasis of NonSmall Cell Lung Cancer by Regulating BMI1. Cell Physiol Biochem 2015;37:387-398.

7 Li J, Yu T, Cao J, Liu L, Liu Y, Kong HW, Zhu MX, Lin HC, Chu DD, Yao M, Yan MX: MicroRNA-148a Suppresses Invasion and Metastasis of Human Non-Small-Cell Lung.Cancer. Cell Physiol Biochem 2015; 37:1847-1856.

8 Raza U, Zhang JD, Sahin 0: Micrornas: Master regulators of drug resistance, stemness, and metastasis. J Mol Med (Berl) 2014;92:321-336.

-9 MacDonagh L, Gray SG, Finn SP, Cuffe S, O'Byrne KJ, Barr MP: The emerging role of micrornas in resistance to lung cancer treatments. Cancer Treat Rev 2015;41:160-169.

10 Wu L, Cai C, Wang X, Liu M, Li X, Tang H: Microrna-142-3p, a new regulator of rac1, suppresses the migration and invasion of hepatocellular carcinoma cells. FEBS Lett 2011;585:1322-1330.

11 Lv M, Zhang X, Jia H, Li D, Zhang B, Zhang H, Hong M, Jiang T, Jiang Q, Lu J, Huang X, Huang B: An oncogenic role of mir-142-3p in human t-cell acute lymphoblastic leukemia (t-all) by targeting glucocorticoid receptor-alpha and camp/pka pathways. Leukemia 2012;26:769-777.

12 Wang XS, Gong JN, Yu J, Wang F, Zhang XH, Yin XL, Tan ZQ Luo ZM, Yang GH, Shen C, Zhang JW: Microrna29a and microrna-142-3p are regulators of myeloid differentiation and acute myeloid leukemia. Blood 2012;119:4992-5004.

13 MacKenzie TN, Mujumdar N, Banerjee S, Sangwan V, Sarver A, Vickers S, Subramanian S, Saluja AK: Triptolide induces the expression of mir-142-3p: A negative regulator of heat shock protein 70 and pancreatic cancer cell proliferation. Mol Cancer Ther 2013;12:1266-1275.

-14 Xu G, Wang J, Jia Y, Shen F, Han W, Kang Y: Mir-142-3p functions as a potential tumor suppressor in human osteosarcoma by targeting hmga1. Cell Physiol Biochem 2014;33:1329-1339.

15 Shen WW, Zeng Z, Zhu WX, Fu GH: Mir-142-3p functions as a tumor suppressor by targeting cd133, abcg2, and lgr5 in colon cancer cells. J Mol Med (Berl) 2013;91:989-1000.

- 16 Colamaio M, Puca F, Ragozzino E, Gemei M, Decaussin-Petrucci M, Aiello C, Bastos AU, Federico A, Chiappetta G, Del Vecchio L, Torregrossa L, Battista S, Fusco A: Mir-142-3p down-regulation contributes to thyroid follicular tumorigenesis by targeting ash1l and mll1. J Clin Endocrinol Metab 2015;100:E59-69.

17 Qi X, Li J, Zhou C, Lv C, Tian M: Mir-142-3p suppresses socs6 expression and promotes cell proliferation in nasopharyngeal carcinoma. Cell Physiol Biochem 2015;36:1743-1752.

18 Xiao P, Liu WL: MiR-142-3p functions as a potential tumor suppressor directly targeting HMGB1 in nonsmall-cell lung carcinoma. Int J Clin Exp Pathol. 2015;8(9):10800-7

19 Boeri M, Verri C, Conte D, Roz L, Modena P, Facchinetti F, Calabro E, Croce CM, Pastorino U, Sozzi G: Microrna signatures in tissues and plasma predict development and prognosis of computed tomography detected lung cancer. Proc Natl Acad Sci USA 2011;108:3713-3718.

20 Kaduthanam S, Gade S, Meister M, Brase JC, Johannes M, Dienemann H, Warth A, Schnabel PA, Herth FJ, Sültmann H, Muley T, Kuner R: Serum mir-142-3p is associated with early relapse in operable lung adenocarcinoma patients. Lung Cancer 2013;80:223-227.

-21 Lei Z, Xu G, Wang L, Yang H, Liu X, Zhao J, Zhang HT: Mir-142-3p represses tgf-beta-induced growth inhibition through repression of tgfbetar1 in non-small cell lung cancer. FASEB J 2014;28:2696-2704.

-22 Avalos Y, Canales J, Bravo-Sagua R, Criollo A, Lavandero S, Quest AF: Tumor suppression and promotion by autophagy. Biomed Res Int 2014;2014:603980.

23 White E, DiPaola RS: The double-edged sword of autophagy modulation in cancer. Clin Cancer Res 2009;15:5308-5316.

24 Livesey KM, Tang D, Zeh HJ, Lotze MT: Autophagy inhibition in combination cancer treatment. Curr Opin Investig Drugs 2009;10:1269-1279.

25 Lee JG, Shin JH, Shim HS, Lee CY, Kim DJ, Kim YS, Chung KY: Autophagy contributes to the chemo-resistance of non-small cell lung cancer in hypoxic conditions. Respir Res 2015;16:138.

26 Tang D, Kang R, Zeh HJ, 3rd, Lotze MT: High-mobility group box 1 and cancer. Biochim Biophys Acta 2010;1799:131-140. 


\section{Cellular Physiology Cell Physiol Biochem 2017;41:1370-1382

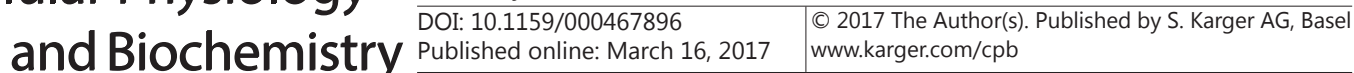

-27 Tang D, Kang R, Cheh CW, Livesey KM, Liang X, Schapiro NE, Benschop R, Sparvero LJ, Amoscato AA, Tracey KJ, Zeh HJ, Lotze MT: Hmgb1 release and redox regulates autophagy and apoptosis in cancer cells. Oncogene 2010;29:5299-5310.

28 Yang L, Yu Y, Kang R, Yang M, Xie M, Wang Z, Tang D, Zhao M, Liu L, Zhang H, Cao L: Up-regulated autophagy by endogenous high mobility group box-1 promotes chemoresistance in leukemia cells. Leuk Lymphoma 2012;53:315-322.

-29 Wang L, Zhang H, Sun M, Yin Z, Qian J: High mobility group box 1-mediated autophagy promotes neuroblastoma cell chemoresistance. Oncol Rep 2015;34:2969-2976.

-30 Pan B, Chen D, Huang J, Wang R, Feng B, Song H, Chen L: Hmgb1-mediated autophagy promotes docetaxel resistance in human lung adenocarcinoma. Mol Cancer 2014;13:165.

- 31 Zhang R, Li Y, Wang Z, Chen L, Dong X, Nie X: Interference with hmgb1 increases the sensitivity to chemotherapy drugs by inhibiting hmgb1-mediated cell autophagy and inducing cell apoptosis. Tumour Biol 2015;36:8585-8592.

32 Bjørkøy G, Lamark T, Brech A, Outzen H, Perander M, Overvatn A, Stenmark H, Johansen T: P62/sqstm1 forms protein aggregates degraded by autophagy and has a protective effect on huntingtin-induced cell death. J Cell Biol 2005;171:603-614.

- 33 Sun H, Wang Z, Yakisich JS: Natural products targeting autophagy via the pi3k/akt/mtor pathway as anticancer agents. Anticancer Agents Med Chem 2013;13:1048-1056.

- 34 Kumar D, Das B, Sen R, Kundu P, Manna A, Sarkar A, Chowdhury C, Chatterjee M, Das P: Andrographolide analogue induces apoptosis and autophagy mediated cell death in u937 cells by inhibition of pi3k/akt/ mtor pathway. PLoS One 2015;10:e0139657.

- 35 Zhao R, Chen M, Jiang Z, Zhao F, Xi B, Zhang X, Fu H, Zhou K: Platycodin-D Induced Autophagy in Non-Small Cell Lung Cancer Cells via PI3K/Akt/mTOR and MAPK Signaling Pathways. J Cancer 2015;6:623-631. White E: The role for autophagy in cancer. J Clin Invest 2015;125:42-46.

- 37 Yang X, Yu DD, Yan F, Jing YY, Han ZP, Sun K, Liang L, Hou J, Wei LX: The role of autophagy induced by tumor microenvironment in different cells and stages of cancer. Cell Biosci 2015;5:14.

38 Rebecca VW, Amaravadi RK: Emerging strategies to effectively target autophagy in cancer. Oncogene 2016;35:1-11.

-39 Ozpolat B, Benbrook DM: Targeting autophagy in cancer management - strategies and developments. Cancer Manag Res 2015; 7:291-299.

40 Hwang KE, Kim YS, Jung JW, Kwon SJ, Park DS, Cha BK, Oh SH, Yoon KH, Jeong ET, Kim HR: Inhibition of autophagy potentiates pemetrexed and simvastatin-induced apoptotic cell death in malignant mesothelioma and non-small cell lung cancer cells. Oncotarget 2015;6:29482-29496.

-41 Sugita S, Ito K, Yamashiro Y, Moriya S, Che XF, Yokoyama T, Hiramoto M, Miyazawa K: Egfr-independent autophagy induction with gefitinib and enhancement of its cytotoxic effect by targeting autophagy with clarithromycin in non-small cell lung cancer cells. Biochem Biophys Res Commun 2015;461:28-34.

42 Wu G, Li H, Ji Z, Jiang X, Lei Y, Sun M: Inhibition of autophagy by autophagic inhibitors enhances apoptosis induced by bortezomib in non-small cell lung cancer cells. Biotechnol Lett 2014;36:1171-1178.

43 Zou Y, Ling YH, Sironi J, Schwartz EL, Perez-Soler R, Piperdi B: The autophagy inhibitor chloroquine overcomes the innate resistance of wild-type egfr non-small-cell lung cancer cells to erlotinib. J Thorac Oncol 2013;8:693-702.

-44 Sui X, Kong N, Zhu M, Wang X, Lou F, Han W, Pan H: Cotargeting egfr and autophagy signaling: A novel therapeutic strategy for non-small-cell lung cancer. Mol Clin Oncol 2014;2:8-12.

45 Wei J, Ma Z, Li Y, Zhao B, Wang D, Jin Y, Jin Y: Mir-143 inhibits cell proliferation by targeting autophagyrelated 2b in non-small cell lung cancer h1299 cells. Mol Med Rep 2015;11:571-576.

46 Yao S, Zhao T, Jin H: Expression of microrna-325-3p and its potential functions by targeting hmgb1 in nonsmall cell lung cancer. Biomed Pharmacother 2015;70:72-79. 